\title{
An Enhanced Difference Method for Multi-objective Model of Cellular Base Station Antenna Configurations
}

\author{
Xiaofei Wang, Zhipeng Jiang, Suixiang Gao \\ School of Mathematical Sciences, University of CAS, Beijing, China \\ Email: wangxiaofei110@mails.ucas.ac.cn, lstjzp07@aliyun.com,sxgao@ucas.ac.cn
}

Received July, 2013

\begin{abstract}
In this paper, we propose a fine-grained grid-based multi-objective model which aims at optimizing base station antennas' configurations, such as transmit power, antenna tilt and antenna azimuth, in order to upgrading network performance in cellular networks. As the model is non-convex, non-smooth and discrete and computationally expensive, we use decomposition method to solve the MOP problem. We mainly focus on addressing the scalarized sub-problem after decomposition. For the scalarized sub-problem, we propose an enhanced difference method. First, difference of each component is calculated, which provides the guidance of optimization. Then an OPSO is applied to search the optimal step length. The method is applied to GSM network optimization on an area in Beijing. The effect of the application shows that proposed method has a good performance, and is effective/efficient to solve mobile network optimization problems.
\end{abstract}

Keywords: Mobile Network Optimization; Multi-objective Optimization; Decomposition, Difference Method, Particle Swarm Optimization

\section{Introduction}

Wireless networks such as GSM, UMTS etc. are based on the same cellular concept. A cellular network is a radio network distributed over land areas called cells, each served by at least one fixed-location transceiver, known as a cell site or base station antenna. Due to the high costs and the scarcity of radio resources, an accurate and efficient mobile network planning appears of outmost importance. With the rapid growth of network size and number of users, efficient quantitative methods to support decisions for base station (BS) location have become essential. This need is now even more acute due to the increased complexity of the system and the number of parameters that must be considered [2]. In order to improve the quality of service (QoS) in cellular networks, it is very important to implement the network planning and optimization in cellular networks. Network planning refers to the process of designing network structure and topology subject to various design requirements. Network optimization amounts to finding a network configuration to achieve the best possible performance.

In order to improve the QOS of a cellular network, some factors have to be considered, e.g. upgrading coverage rate, decreasing interference, increasing coverage continuity and enhancing cells' traffic equilibrium. In real system planning, optimizing BS configuration can often be more critical than BS location since service providers may have a very limited set of candidate sites due to authority constraints on new antenna installation and on electromagnetic pollution in urban areas. So they are usually very interested in optimizing the QOS with the available BSs by adjusting their configurations, adding new BSs only when it is strictly necessary.

In detailed planning, making major changes in network topology and layout is typically not an acceptable option for an operator. Instead, the goal is to optimize antennas' key configuration parameters [7]. Meanwhile, the optimization of every one of these parameters is affected by the other parameters and other antennas. Because of this, it is difficult and even impossible to optimize the three configuration parameters by a manual approach. Automated network planning and optimization through mathematical model and algorithms allow operators to better deal with the complexity of cellular network optimization, a task that is often beyond the reach of a manual approach. In addition to making the network optimization process time-efficient, planning tools incorporating automated optimization can significantly reduce network deployment and maintenance costs.

There have been many works on cellular networks planning and optimization. Almost all the previous works establish a model with a discrete solution space and 
based on few test points. But as mentioned in [8], the optimization conducted on few test points can't ensure the same good performance for other region. Besides, in order to improve the QoS of a cellular network, some objectives/factors have to be considered simultaneously, e.g. upgrading coverage rate, decreasing interference, increasing coverage continuity and enhancing cells' traffic equilibrium. These factors or objectives are often dependent and conflict. For instance, decreasing the transmit power reduce the cell overlap and interference. On the other hand, coverage problems will arise if the transmit power becomes too low. So, a multi-objective optimization problem should be considered to improve the QoS of mobile network. In this paper, we propose a multi-objective optimization model of QOS and base station antenna configurations with fine-grained grid division developed in [8], and an enhanced difference method is designed for the proposed MOP.

This paper is organized as follows. Section 2 discusses the cellular networks planning and optimization problems on previous work. In Section 3, a multi-objective optimization model of QOS and base station antenna configurations is proposed in cellular networks. In Section 4, we give the enhanced difference method to solve the proposed MOP model. Computational results obtained with realistic instances are reported and discussed in Section 5. The paper is then concluded in Section 6.

\section{Previous Work}

Many previous works have discussed the optimization of the location and configuration of base stations.

In [2], the authors proposed discrete optimization models which consider the signal-to-interference ratio as quality measure and algorithms aimed at supporting the decisions in the process of planning where to locate new BSs. The similar methods are arisen in [3-5], [15]. But all these studies are about the position on which the BSs are installed without considering the adjustment of the antennas' configuration. In [6] the authors gave a basic model for pilot power optimization subject to a full coverage constraint as well as its extended version which considers various coverage levels and to consider user traffic distribution over the network. In [7] automated optimization of service coverage and radio base station antenna configuration is addressed and three key configuration parameters: transmit power of the common pilot channel (CPICH), antenna tilt and antenna azimuth are considered. The optimization target is minimizing the total CPICH power under the constraint that every bin (Each bin is a test point) is in the coverage area of at least one cell. In [9-11], a mathematical mixed-integer programming model for planning cost-efficient radio networks under network quality constraints and some net- work planning methods based on this model are presented.

All these studies suppose that a little area with a given amount of traffic can be considered as a test point and the whole optimization area is denoted by all this kind of points. Meanwhile, all these studies give models with constraints like that the signal received by all the test points must be under the given constraints (i.e. the signal strength received by every point have to be strong enough, and the signal to interference ratio of every point must be greater than a given threshold.). Then they optimized some other performance parameters (e.g. minimizing the cost or total transmit power) with this constraint and some other constraints. But in real application, constraints like that the signal received by all the test points must be under the given constraints may not be satisfied no matter how the antennas' configuration of the network being set.

In [12] various parameters of cellular base station (BS) placement problem such as site coordinates, transmit power, height and tilt angle are determined using evolutionary multi-objective algorithm to obtain better compromised solutions. And the maximization of service coverage and minimization of cost are considered as conflicting objectives by satisfying inequality constraints such as handover, traffic demand and overlap. In [13] the authors address the design of the network which has been formulated as a multi-objective constrained combinatorial optimization problem and propose a genetic algorithm that aims to approximate the Pareto frontier of the problem. But the detailed formulas of the objectives are not given in these studies.

In [14] the authors address the problem of capacity optimization in a Universal Mobile Telecommunication System (UMTS) radio network and present an optimization algorithm for finding the best settings of the antenna tilt and common pilot channel power of the base stations. But only an algorithm is presented in this paper without any model.

\section{A Multi-objective Model of QoS and Base Station Antenna Configurations}

In the section, A multi-objective optimization model is proposed.

As mentioned in introduction section, there are several indicators that reflect performance of the network, such as interference, coverage rate, the connectivity of primary service area and traffic balance. In this paper, the objectives we consider are coverage rate, interference and the connectivity of primary service, and the variables are antenna's azimuth, tilt and transmit power. Traffic balances are considered as constraints, because the distribution of grid traffic varies from time to time, it's very 
difficult to handle all cases. In order to optimize a rectangle region by adjusting some antennas' parameters, the region surrounding must be considered. Otherwise, after optimization, the objectives of region get improved but the region surrounding may get worse. Besides considering the optimization region, we also take account of the region around as protect region.

The main goal is to optimize the objectives of optimization region by adjusting antennas' parameter and meanwhile to avoid the objectives of protect regions getting worse. In addition, antennas must not be overloaded. The antennas selected to be adjusted are determined in advance.

\subsection{Multi-objective Model}

Consider a rectangle optimization region $Z$ and corresponding protect region $Z^{\prime}$, divided into $I$ and $J$ grids with identical or different size respectively. We assume the strength of the received signal from an antenna at all the points in a grid is identical and the total traffic of every grid is received only by one antenna. In order to make the hypothesis be rational, the grid size have to be small enough. Suppose there are $A K$ antennas that can affect the region $Z$ and $Z^{\prime}$. An antenna affect a region if the strength of the received signal in at least one grid in the region from this antenna is greater than a given threshold (e.g. $-104 \mathrm{dBm}$ ) and the strength of the received signal of all the grids from each antenna can be estimated according to empirical propagation models such as Hata and COST-231 or to more precise but computationally intensive ray tracing techniques. Let the strength of the received signal in grid $i$ from antenna $k$ be $P_{i k}$. If the strength of the received signal in grid $i$ from antenna $k$ is the strongest in all antennas which can affect the grid $i$, then antenna $k$ is called the master antenna of grid $i$ and the strength of the received signal from antenna $k$ is called the master strength. Let $N_{i}$ be the geographic neighbor of grid $i$. The antenna $k_{i}$ is the master antenna of grid $i$.

The proposed multi-objective optimization model ( $M O P$ ) is given below: $R$ is a very large number. $K$ is set of adjusted antennas. $T K$ is the set of antennas, traffic balance of which are considered. $t_{u}$ represents the traffic of grid $u$. $P_{\text {target }}$ is strength threshold. $A K$ contains $K$ and $T K$. $A K$ and $T K$ are determined by $K$ and propagation model. $T T_{k}$ is total traffic received by antenna $k . T\left(x_{0}\right)$ and $T(x)$ represent the traffic received from optimization region and protect region under the adjusted antenna's configure $x_{0}$ and $x . M L_{k}$ is the maximum traffic load of antenna $k$, which is determined by the number of antenna's frequency channels and maximum capacity of per channel. $x_{0}$ is the existing antennas' configuration of the network.

$$
\begin{array}{ll}
\min _{x} & f(x)=\left(f_{1}(x), f_{2}(x), f_{3}(x)\right)^{T} \\
\text { s.t. } & h_{u} \cdot R \geq P_{\text {target }}-P_{u k_{u}}(x), \quad \forall u \in I \cup J \\
& g_{u} \geq\left|9 k_{u}-\sum_{v \in N_{v}} k_{v}\right| / R, \quad \forall u \in I \cup J \\
& k_{u}=\arg \max _{k \in A K} P_{u k}(x), \quad \forall u \in I \cup J \\
& \sum_{i \in I} h_{i} \leq W_{0} \\
& \sum_{i \in I}\left(P_{i k_{i}}(x) / \sum_{k \in A k} P_{i k}(x)\right) \geq E_{0} \\
& \sum_{i \in I} g_{i} \geq B_{0} \\
& \sum_{j \in J} h_{j} \leq W_{0}^{\prime} \\
& \sum_{j \in J}\left(P_{j k_{j}}(x) / \sum_{k \in A k} P_{j k}(x)\right) \geq E_{0}^{\prime} \\
& \sum_{j \in J} g_{j} \geq B_{0}^{\prime} \\
& T T_{k}-T_{k}\left(x^{0}\right)+T_{k}(x) \leq M L_{k}, \quad \forall k \in T K \\
& x \in X
\end{array}
$$

where

$$
\begin{aligned}
f_{1}(x) & =\frac{1}{|I|} \sum_{i \in I} h_{i} \\
f_{2}(x) & =\sum_{i \in I}\left(1-\left(P_{i k_{i}}(x) / \sum_{k \in A K} P_{i k}(x)\right)\right)^{2} \\
f_{3}(x) & =\frac{1}{|I|} \sum_{i \in I} g_{i} \\
T_{k}(x) & =\sum_{\left\{u \in I \cup J \mid k_{u}=k\right\}} t_{u}
\end{aligned}
$$

$W_{0}, E_{0}, B_{0}, W_{0}^{\prime}, E_{0}^{\prime}, B_{0}^{\prime}$ are thresholds that represent the objective function values of optimization region and protect region under the existing antenna configuration of the network respectively.

\subsection{Variables, Objectives and Constraints}

Three key base station antenna configuration parameters, transmit power, antenna tilt and antenna azimuth, are considered in this model. Then the decision variable $x$ is set as $x=\left(\alpha_{1}, \beta_{1}, p_{1}, \cdots, \alpha_{K}, \beta_{K}, p_{K}\right)^{\prime}$, where $\alpha_{i}$ is the antenna $i^{\prime}$ azimuth, and $\beta_{i}$ is antenna tilt, $p_{i}$ is transmit power. $X$ is the rectangle boundary set of $x$. As mentioned in [1], each antenna is configured by some engineering parameters and the adjustment of every configuration parameters is limited in a special range.

There are three objective functions in this model. First objective is to minimize the weak coverage rate of optimization region $Z$. If the master strength of grid $i$ is less than a given threshold $P_{\text {target }}$ (i.e. $-90 \mathrm{dBm}$ ), the grid $i$ is called weak-covered grid because master strength is too weak to be received by receivers. Second objective is to minimize the interference from other antenna. We evalu- 
ate this objective by considering the ratio of interference energy and total receive energy. The third objective is to reduce the boundary grid rate, which is to decrease the switching possibility. When a calling moves from a grid in which the master antenna is antenna $k$ to a grid in which the master antenna is antenna $q$ and $k \neq q$, the access antenna of this calling may switch from $k$ to $q$. The dropped possibility of the calling is increasing during the process of switching. So in order to decreasing the call dropping possibility caused by the switching, the rate of cell boundary grids has to be reduced.

Constrains (4)-(6) represent that optimal solution must outperform the existing configuration in terms of optimization region. Constrains (7)-(9) represent that the objectives of protect region must not get worse after optimization. Constrains (10) represent that antenna traffic must not be exceed maximum load. In practical optimization, objectives in optimization region may conflict with the one in protect region. And if we keep the constraint strictly satisfied, there may be no feasible solution. Thus, in this paper, we consider the relaxation form of MOP and get $\varepsilon-M O P$. For the sake of saving space, the right terms in constraints (7)-(9) are multiplied by $(1+\varepsilon)$. If $\varepsilon=0, \varepsilon-M O P$ and $M O P$ are the same.

\section{Enhanced Difference Method}

The MOP is difficult to solve because it is nonconvex nonsmooth and discrete. And the function evaluation is computationally expensive, so we choose a decomposition-based multi-objective optimization method. By decomposition, the MOP is transformed into single objective problem. Thus, the classical single optimization methods are used to solve it in a fast convergence. In this paper, we use the popular decomposition-based framework-MOEA/D [16] to decompose the MOP. In this paper, we mainly consider approaches for subproblems, which are taken as an option in [16]. The mail loop in MOEA/D is used to help subproblems jump out of local optimal.

As the sub-problem is also a nonconvex, nonsmooth and discrete, it's difficult to solve it by classical gradient-based methods. We use the difference instead of gradient to guide the search, but the difference is used in a different way, no the same with coordinate rotation method. Thus, an enhanced difference method is proposed. First the penalty NBI method is applied to transform the MOP into single objective function. Then the difference of every component is calculated. After that component difference is modified into improved difference matrix (Algorithm 2). In the last, we use OPSO [17] to get the step length with the guide of improved difference matrix (Algorithm 1);

We list the proposal method -- Enhanced Difference Method below:

\section{Algorithm 1 Enhanced Difference Method}

Input: weight vector $\lambda$, reference point $z^{*}$, penalty parameter $\theta$

Output: optimal solution

Step 1. Initiate first solution $x^{t}=x^{0}, t=0$

Step 2. Use Penalty NBI method, scalarize the MOP

$$
\begin{aligned}
& g\left(x^{t}\right)=d_{1}+\theta \cdot d_{2}, d_{1}=\frac{\left\|\left(f(x)-z^{*}\right)^{T} \lambda\right\|}{\|\lambda\|} \\
& d_{2}=\left\|f(x)-\left(z^{*}+d_{1} \lambda\right)\right\|
\end{aligned}
$$

Step 3 Calculate improved difference matrix $D^{t}$ of , see Algorithm 2

Step 4. Apply OPSO to search the best step size array for improved difference of current solution $\mathrm{x}$, which is to solve the following optimization:

$$
\begin{array}{ll}
\min _{\alpha^{t}} & g\left(x^{t}+\left(\alpha^{t}\right)^{T} D_{k}\right) \\
\text { s.t. } & 0 \leq \alpha_{i}^{t} \leq u_{i} \\
& \alpha_{i}^{t} \in N
\end{array}
$$

where $u_{i}$ is max step size for dimension $i, D^{t}$ is the improved difference matrix.

Step 5. let $x^{t+1}=x^{t}+\left(\alpha^{t}\right)^{T} D_{k}, t:=t+1$. If stopping condition is satisfied, then stop and output $x^{t}$. Otherwise, go to Step 2.

\section{Algorithm 2 Improved Difference Matrix \\ Input: a scalarized function $g\left(x^{t}\right)$ \\ Output: improved difference matrix $D^{t}$}

Step 1. Initialize $D^{t}=0$, and initialize max difference matrix $G^{t}=0$.

Step 2. Calculate each component's forward difference and backward difference:

$$
g_{i}^{+}=\frac{g\left(x^{t}+\delta_{i} \cdot e_{i}\right)-g\left(x^{t}\right)}{\delta_{i}}, g_{i}^{-}=\frac{g\left(x^{t}\right)-g\left(x^{t}-\delta_{i} \cdot e_{i}\right)}{\delta_{i}}
$$

where $\delta_{i}$ is the minimum step length of component $i$.

Step 3. Calculate forward improved value and backward improved value

$$
p_{i}=\max \left(0,-g_{i}^{+}\right), n_{i}=\max \left(0, g_{i}^{-}\right)
$$

Set $G_{i i}=p_{i}$. if $n_{i} \geq p_{i}$, let $G_{i i}=p_{i}$.

Step 4. for each component $i$, set $D_{i i}=\operatorname{sgn}\left(G_{i i}\right) \cdot \delta_{i}$, $\operatorname{sgn}(x)$ is sign function.

In next section, we validate the proposal method with real operational network.

\section{Simulation and Application}

In this section, we apply our model and algorithm on the GSM1800 networks of China Mobile Communications Corporation. The implementation is on a region in Beijing. The area of the optimization region $Z$ is $1.1 \mathrm{~km} \times$ $1.7 \mathrm{~km}$ and the area of protect region is $7.5 \mathrm{~km} \times 7.9 \mathrm{~km}$. 
The grid size is $5 m \times 5 m$. There are 30 antennas are selected to be adjusted. The propagation model used is a new high-precision propagation model in our previous work [8]. The average error of the prediction of the field strength is lower than $6.5 \mathrm{dBm}$. Our work is supported by China Mobile Group Beijing Limited Company, and the data of base station antennas' configuration and the electronic map are provided by them. We execute our work on a DELL server with 32G memory and 8 CPUs. The algorithm is implemented by $\mathrm{VC}++$.

The transmit power is set in the range $[29 \mathrm{dBm}$, $43 \mathrm{dBm}$ ], minimum step length is $2 \mathrm{dBm}$. The maximum change of Azimuth to the left direction is set to $40^{\circ}$, to the right direction is set to $35^{\circ}$, minimum step length is $5^{\circ}$. The tilt is in the range $\left[0^{\circ}, 30^{\circ}\right]$, and minimum step length is $2^{\circ}$. The max step sizes in each iteration for all components are same to 5 , that is $u_{i}=5$. And we set $\varepsilon=0.05$.

We select 4 different weight vectors to test the method. They are $(1,0,0),(0,1,0),(0,0,1),(1 / 3,1 / 3,1 / 3)$. The total computation time is about 1 hour, and the optimization results are as follows. The functions values listed in the following tables are the ratio between real function values and function values of $x^{0}$, that is

$$
f_{i}^{\prime}(x)=f_{i}(x) / f_{i}\left(x^{0}\right), \quad i=1,2,3 .
$$

The results obtained by enhanced difference method corresponding to the 4 weight vectors are listed in Table 1. We take weight vector $(0,1,0)$ as example, weak coverage rate after optimization is reduced by $11 \%$ and interference energy is reduced by $20 \%$ and boundary grid rate is reduced by $11 \%$. So after applying the proposed method, the performance of GSM1800 network is improved significantly. The result shows the method is effective and efficient.

The adjusted result of the antennas' configuration with weight vector $(0,1,0)$ is listed in Table 2 . The ID is antenna's identifier. The $\mathrm{Ab}$ and Aa represent the azimuth before and after optimization respectively. The Tb and Ta represent the tilt before and after optimization respectively. The $\mathrm{Pb}$ and $\mathrm{Pa}$ represent the transmit power before and after optimization respectively. The blank grid represents that this type of configuration of the antenna remain unchanged after optimization.

Table 1. The relative objective values of GSM1800 after optimization.

\begin{tabular}{cccc}
\hline$\lambda$ & $f_{1}^{\prime}(x)$ & $f_{2}^{\prime}(x)$ & $f_{2}^{\prime}(x)$ \\
\hline$(0,0,1)$ & 0.93 & 0.81 & 0.94 \\
$(0,1,0)$ & 0.89 & 0.8 & 0.89 \\
$(1,0,0)$ & 0.95 & 0.85 & 0.96 \\
$(1 / 3,1 / 3,1 / 3)$ & 0.89 & 0.81 & 0.93 \\
\hline
\end{tabular}

Table 2. The adjusted result.

\begin{tabular}{ccccccc}
\hline ID & Ab & Aa & $\mathrm{Tb}$ & $\mathrm{Ta}$ & $\mathrm{Pb}$ & $\mathrm{Pa}$ \\
\hline 30114 & 40 & 20 & 6 & 22 & 43 & 39 \\
30115 & 205 & 185 & & & & \\
30303 & & & & & 39 & 43 \\
$\ldots$ & $\ldots$ & $\ldots$ & $\ldots$ & $\ldots$ & $\ldots$ & $\ldots$ \\
33153 & 180 & 150 & & & 43 & 31 \\
$\ldots$ & $\ldots$ & $\ldots$ & $\ldots$ & $\ldots$ & $\ldots$ & $\ldots$ \\
34398 & & & 3 & 25 & 43 & 33 \\
34399 & 300 & 270 & 3 & 5 & 43 & 33 \\
\hline
\end{tabular}

\section{Conclusions}

In this paper, we propose a fine-grained grid based multi-objective model which aims at optimizing base station antennas' configurations, such as transmit power, antenna tilt and antenna azimuth, in order to upgrading network performance in cellular networks. As the model is nonconvex, discrete and computationally expensive, we use decomposition method MOEA/D to solve the MOP problem. We mainly study the optimization of the subproblem in MOEAD framework. We propose an enhanced difference method to solve these subproblems. First, Penalty NBI scalarization method is used to transform MOP to single objective optimization. Second, OPSO is coupled with difference method to find best step size to minimize the scalarized objective. The improved difference is used only to find which dimension could be minimized; the real step length in each iteration is generated by OPSO method. We apply the MOP model and algorithm to optimize GSM1800 network on a region which has bad performance in YuQuanlu district in Beijing, collaborated with the China Mobile Group Beijing Limited Company. The application results show that the network performance in the region is greatly improved after optimization. The Model and algorithm are effective and efficient for real network optimization.

\section{Acknowledgements}

The authors would like to express their sincere thanks to CMCC and Engineers working in China Mobile Group Guangdong Company Guangzhou Branch and Beijing Limited Company for their kindly suggesting the problem, providing the data, and for their invaluable support.

\section{REFERENCES}

[1] H. Meunier, E. Talbi and P. Reininger, "A Multiobjective Genetic Algorithm for Radio Network Optimization,” Proc. Congress on Evolutionary Computation, Vol. 1, 2000, pp. 317-324. 
[2] E. Amaldi, A. Capone and F. Malucelli, "Planning UMTS Base Station Location: Optimization Models with Power Control and Algorithms," IEEE Transactions on Wireless Comm, Vol. 2, No. 5, 2003, pp. 939-952. doi:10.1109/TWC.2003.817438

[3] E. Amaldi, A. Capone, F. Malucelli and F. Signori, "Optimization Models and Algorithms for Downlink UMTS Radio Planning," Proc. IEEE Wireless Communications and Networking Conf, New Orleans, 20-20 Mar 2003, pp. 827-831.

[4] E. Amaldi, A. Capone and F. Malucelli, "Improved Models and Algorithms for UMTS Radio Planning," Proceedings of IEEE VTC Fall 2001. Atlantic City, 7-11 Oct 2001, pp. 920-924.

[5] E. Amaldi, A. Capone and F. Malucelli, "Optimizing Base Station Siting in UMTS Networks," Proceedings of IEEE VTC Spring 2001, Rhodes, 6-9 May 2001, pp. 2828-2832.

[6] I. Siomina and D. Yuan, "Pilot Power Management in WCDMA Networks: Coverage Control with Respect to Traffic Distribution," Proc. Seventh ACM International Symposium on Modeling, Analysis and Simulation of Wireless and Mobile Systems (MSWiM), New York, 2004, pp. 276-282.

[7] I. Siomina, P. Varbrand and D. Yuan, "Automated Optimization of Service Coverage and Base Station Antenna Configuration in UMTS Networks," IEEE Wireless Communications Magazine. Vol. 13, No. 6, 2006, pp. 16-25. doi:10.1109/MWC.2006.275194

[8] T. Guo, S. Gao, et al., "High Precision Coverage Optimization Models and Algorithms for GSM and TD-SCDMA Networks,” IFORS 2011, Melbourne, July 2011.

[9] A. Eisenblatter, A. Fugenschuh, H. Geerdes, D. Junglas, T. Koch and A. Martin, "Integer Programming Methods for UMTS Radio Network Planning,” Proc. of WiOpt'04, Cambridge, UK, 2004.

[10] A. Eisenblatter, T. Koch, A. Martin, T. Achterberg, A. Fugenschuh, A. Koster, O. Wegel and R. Wessaly,
"Modelling Feasible Network Configurations for UMTS," In: G. Anandalingam, S. Raghavan, Ed., Telecommunications Network Design and Management, Springer US, 2003, pp. 1-23. doi:10.1007/978-1-4757-3762-2_1

[11] A. Eisenblatter, E. Fledderus, A. Fugenschuh, H. Geerdes, B. Heideck, D. Junglas, T. Koch, T. Kurner and A. Martin, "Mathematical Methods for Automatic Optimization of UMTS Radio Networks,” Tech. Rep. D43, IST-2000-28088 Momentum , 2003.

[12] N. Lakshminarasimman, S. Baskar, A. Alphones and M. WilljuiceIruthayarajan, "Evolutionary Multiobjective Optimization of Cellular Base Station Locations Using Modified NSGA-II,” J Wirel Networks, Vol. 17, No. 3, 2011, pp. 597-609. doi: 10.1007/s11276-010-0299-2

[13] H. Meunier, E. Talbi and P. Reininger, "A Multiobjective Genetic Algorithm for Radio Network Optimization," Proc. Congress on Evolutionary Computation, La Jolla, 16-19 Jul 2000, pp. 317-324.

[14] A. Gerdenitsch, M. Toeltsch, S. Jakl and Y. Chong, “A Rule Based Algorithm for Common Pilot Channel and Antenna Tilt Optimization in UMTS FDD Networks," ETRI Journal. Vol. 26, No. 5, 2004, pp. 437-442. doi:10.4218/etrij.04.0703.0007

[15] F. Gu, H. Liu and M. Li, "Evolutionary Algorithm for the Radio Planning and Coverage Optimization of 3G Cellular Networks," International Conference on Computational Intelligence and Security, Beijing, 11-14 Dec 2009, pp. 109-113.

[16] Q. Zhang and H. Li, "MOEA/D: A multiobjective Evolutionary Algorithm Based on Decomposition,” Evolutionary Computation, IEEE Transactions on, Vol. 11, No. 6, 2007, pp. 712-731. doi:10.1109/TEVC.2007.892759

[17] H. Wang, H. Li, Y. Liu, C. Li and S. Zeng, "Opposition-based Particle Swarm Algorithm with Cauchy Mutation," Evolutionary Computation, 2007. CEC 2007. IEEE Congress on, Singapore, 25-28 Sept 2007, pp. 4750-4756. 